\title{
High-power laser beam shaping by inseparable two-dimensional binary-phase gratings for surface modification of stamping dies
}

\author{
Shao-Xia Li, Gang Yu* , Cai-Yun Zheng, Xiu-Bo Liu \\ Laboratory for Laser Intelligent Manufacturing, Institute of Mechanics, Chinese Academy of Sciences, 15 Beisihuanxi Road, Beijing 100080, PR China
}

\section{A R T I C L E I N F O}

\section{Article history:}

Received 6 May 2007

Received in revised form

5 March 2008

Accepted 5 March 2008

\section{Keywords:}

Binary-phase gratings

Beam shaping

Diffraction

Laser surface modification

\begin{abstract}
A B S T R A C T
For surface modification of stamping dies, an inseparable two-dimensional binary-phase gratings is introduced to implement the wavefront transformation of high-power laser beams. The design and fabrication of the gratings are described in detail. Two-dimensional even sampling encoding scheme is adopted to overcome the limitations of conventional Dammann grating in the design of twodimensional output patterns. High diffractive efficiency $(>70 \%)$ can be achieved through the transformation of the Gaussian laser beam into several kinds of two-dimensional arrays in focal plan. The application of the binary-phase gratings in the laser surface modification of ductile iron is investigated, and the results show that the hardness and the wear resistance of the sample surface were improved significantly by using the binary-phase gratings.
\end{abstract}

(c) 2008 Elsevier Ltd. All rights reserved.

\section{Introduction}

High-power laser beams are now used extensively in manufacturing. Material-processing applications often require beam shaping to get laser intensity distributions differ significantly from those generated directly by conventional industrial lasers $[1,2]$. Laser surface modification has been used in industrial applications, as it prevents advance abrasion or cracks of the surface. The mechanical properties, especially the wear resistance of the stamping dies is very important in automobile manufacturing. Ductile iron is an attractive kind of material for stamping dies of automobiles because of low cost, good castability and mechanical properties. And the laser beam used in laser surface modification of ductile iron processes is always the original Gaussian spots [3]. The significant improvement in wear resistance by laser surface remelting is at the expense of the excellent self-lubricant, damping properties of graphite, which are as important as the abrasive wear properties in some service conditions. Thus, it would be attractive if the modification layer of the ductile iron contains periodic gradient zone, i.e., the laser remelted zone and the laser transformation hardened zone are formed alternately. In this paper, the original Gaussian laser beam is shaped into two-dimensional patterns of equal-intensity spots with high efficiency.

It is usually difficult to achieve the above transformations by conventional optical elements due to their inherent optical

\footnotetext{
* Corresponding author. Tel./fax: +861082544250.

E-mail address: gyu@imech.ac.cn (G. Yu).
}

properties, and the diffractive efficiency of computational holographic optical elements is low [4]. With the flexibility and high efficiency in laser beam shaping and transformation, binary optical elements (BOEs) are successfully employed to convert the laser beam into many different shapes [5-8]. And these array spots can be realized by using a fractional Talbot imaging setup [9] or a Dammann grating [10]. The former device can be very light efficient (close to 100\%) and compact but it has a major drawback: to achieve good array uniformity, a Gaussian laser beam must be converted into an incident flattop beam with a plan phase front. To achieve high conversion efficiency, two optical components are needed, which reduce the overall efficiency and greatly increase the dimensions of the space-variant array spots. Dammann gratings are space invariant Fourier-domain array generators, which concern nothing with the input beam shape. But the diffractive efficiency is hard to increase due to the separable coding technology of the Dammann gratings. In theory, Vasara introduced inseparable two-dimensional gratings [11] to improve the conversion efficiency of the Dammann gratings. But according to the knowledge of the authors, very few reports are available up to now on the research of high-power laser beam shaping by inseparable two-dimensional binary-phase gratings, which could make the laser beam present array distribution within the spot area and the corresponding application in surface hardening.

In this paper, an inseparable two-dimensional binary-phase grating is presented, which could convert the Gaussian laser beam into several kinds of two-dimensional uniform-intensity array spots with high efficiency. The design of the binary optical elements is discussed and the fabrication method is described. Moreover, the application of the binary optical elements in laser 
surface modification of ductile iron and the corresponding experiment results are presented.

\section{The design and fabrication of the inseparable binary-phase gratings}

The experimental optical system is shown in Fig. 1. A collimated laser beam illuminates the binary-phase grating located in front of the lens. The surface to be hardened is put in the rear focal plane, where the shaped intensity distribution of the laser beam was obtained. The design and fabrication of the binaryphase grating are shown in what follows.

\subsection{The design of the inseparable binary-phase gratings}

In laser material processing, the diffraction efficiency of the laser beam transmission elements should be high enough to ensure high-power laser beam output. Moreover, according to certain surface modification process, different shapes of laser beam are needed, i.e., the design of the binary-phase gratings should be flexible enough to obtain various laser beam shapes. However, the diffractive efficiency and the flexibility of the Dammann grating are limited due to its one-dimensional encoding method. Common examples of possible phase encodings are pixellated and trapezoidal phase encodings, arbitrary coding scheme and the separable Dammann grating [11]. In this paper, the two-dimensional even sampling coding schemes (Fig. 2) was adopted, which is more flexible than separable conventional Dammann gratings and easier than trapezoidal and arbitrary coding scheme.

According to scalar diffraction theory, the Fraunhofer intensity diffraction at the output plane should be

$I(x, y)=\left(\frac{1}{\lambda z}\right)^{2}\left|\mathscr{F}\left\{t\left(x_{0}, y_{0}\right)\right\}\right|^{2}$

where $\lambda$ is the wavelength of the laser, $z$ is the focal length of the lens, and $t\left(x_{0}, y_{0}\right)$ is transmission function of the grating. Assuming that the complex amplitude transmittance function is $t_{0}\left(x_{0}, y_{0}\right)$, and the size of the element is infinity, the complex amplitude transmittance function $t\left(x_{0}, y_{0}\right)$ of the rectangle aperture grating could be expressed as

$t\left(x_{0}, y_{0}\right)=t_{0}\left(x_{0}, y_{0}\right) \frac{1}{d} \operatorname{comb}\left(\frac{x_{0}}{d}\right) \frac{1}{d} \operatorname{comb}\left(\frac{y_{0}}{d}\right)$

where $d$ is the width of a period of the grating, from the convolution of theorem, the spectrum of the grating can be

$T\left(f_{x}, f_{y}\right)=T_{0}\left(f_{x}, f_{y}\right) \operatorname{comb}\left(d f_{x}\right) \operatorname{comb}\left(d f_{y}\right)$

if and only if $x=m \lambda z / d, y=n \lambda z / d(m, n=0, \pm 1, \ldots)$,

$T\left(f_{x}, f_{y}\right) \neq 0$, and $T\left(f_{x}, f_{y}\right)=T_{0}\left(f_{x}, f_{y}\right)$

where $T_{0}\left(f_{x}, f_{y}\right)$ is the spectrum of a single period. The phase of a period in the uniform sampling rectangle aperture grating is

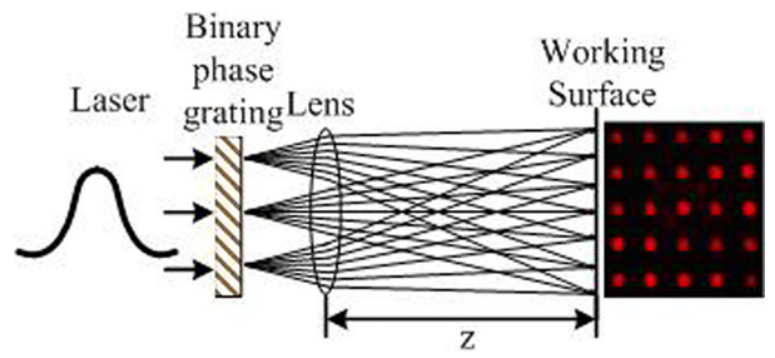

Fig. 1. Experimental arrangement for laser beam shaping and application. composed of gridding aperture cells. Assign each cell with 0 (white cells) or 1 (black cells), which represent that the phase of cell is $\phi_{1}$ and $\phi_{2}$, respectively (Fig. 2). If there are $L$ cells, of which the phase delays are $\phi_{2}$, and the coordinate of the lth cell's nearest apex to the origin is $\left(x_{l}, y_{l}\right)$. From the linearity theorem of Fourier transform, the transmittance function $t_{0}\left(x_{0}, y_{0}\right)$ is

$$
\begin{aligned}
& t_{0}\left(x_{0}, y_{0}\right)=\left[\exp \left(\mathrm{j} \phi_{2}\right)-\exp \left(\mathrm{j} \phi_{1}\right)\right] \sum_{l=1}^{L} \operatorname{rect}\left(x_{0}-\left(x_{l}+\frac{1}{2}\right),\right. \\
& \left.y_{0}-\left(y_{l}+\frac{1}{2}\right)\right)+\exp \left(\mathrm{j} \phi_{1}\right)
\end{aligned}
$$

Then, the spectrum in the output plane can be expressed as

$$
\begin{aligned}
T_{0}\left(f_{x}, f_{y}\right)= & {\left[\exp \left(\mathrm{j} \phi_{2}\right)-\exp \left(\mathrm{j} \phi_{1}\right)\right] \operatorname{sinc}\left(f_{x}\right) \operatorname{sinc}\left(f_{y}\right) \sum_{l=1}^{L} \exp \{-\mathrm{j} 2 \pi} \\
& \left.\times\left[f_{x}\left(x_{l}+\frac{1}{2}\right)+f_{y}\left(y_{l}+\frac{1}{2}\right)\right]\right\}+\exp \left(\mathrm{j} \phi_{1}\right) \delta\left(f_{x}, f_{y}\right)
\end{aligned}
$$

The intensity distribution can be obtained by integrating Eqs. (5) and (1):

$$
\begin{aligned}
I_{0,0} & =I(0,0)=\left(\frac{A}{\lambda z}\right)^{2}\left|\left[\exp \left(\mathrm{j} \phi_{2}\right)-\exp \left(\mathrm{j} \phi_{1}\right)\right] L+\exp \left(\mathrm{j} \phi_{1}\right)\right|^{2} \\
I_{+m,+n} & =I_{-m,-n} \\
& =\left(\frac{A}{\lambda z}\right)^{2}\left|\exp \left(\mathrm{j} \phi_{2}\right)-\exp \left(\mathrm{j} \phi_{1}\right)\right|^{2} \operatorname{sinc}^{2}\left(\frac{m}{d}\right) \operatorname{sinc}^{2}\left(\frac{n}{d}\right) \\
& \times\left|\sum_{l=1}^{L} \exp \left\{-\mathrm{j} 2 \pi\left[\frac{m}{d}\left(x_{l}+\frac{1}{2}\right)+\frac{n}{d}\left(y_{l}+\frac{1}{2}\right)\right]\right\}\right|^{2} \\
I_{-m,+n} & =I_{+m,-n} \\
& =\left(\frac{A}{\lambda z}\right)^{2}\left|\exp \left(\mathrm{j} \phi_{2}\right)-\exp \left(\mathrm{j} \phi_{1}\right)\right|^{2} \operatorname{sinc}^{2}\left(\frac{m}{d}\right) \operatorname{sinc}^{2}\left(\frac{n}{d}\right) \\
& \times\left|\sum_{l=1}^{L} \exp \left\{-\mathrm{j} 2 \pi\left[\frac{m}{d}\left(x_{l}+\frac{1}{2}\right)-\frac{n}{d}\left(y_{l}+\frac{1}{2}\right)\right]\right\}\right|^{2}
\end{aligned}
$$

The elements designed are binary phase, and $\phi_{1}=0, \phi_{2}=\pi$, which represent that the phase of cell is 0 and $\pi$, respectively. The diffraction efficiency and the uniformity of the binary-phase

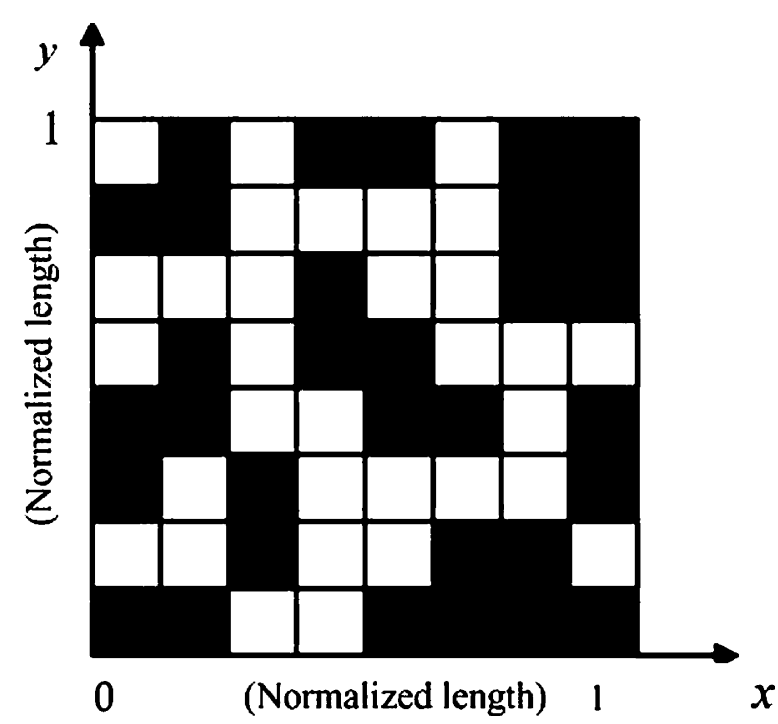

Fig. 2. Coding schemes for pixellated grating (the phase distribution of one period of the binary optics with white cells for 0 phase delay and black cells for $\pi$ phase delay). 
elements are defined by

$\eta=\sum_{\substack{m=0 \\ n=0}}^{N} I_{m, n} / I_{0} \quad$ uni $=\sum_{\substack{m=0 \\ n=0}}^{N}\left(I_{m n}-\bar{I}\right) / I_{0}$.

where the series is summed over only those points that form the uniform-intensity array, and $\bar{I}$ is the average intensity of these points, and $I_{0}$ is the input intensity of the laser beam.

The simulation anneal algorithm [12-14] is adopted to optimize the result, and several two-dimensional array spots are obtained, such as the $5 \times 5$ array spot (Fig. 3b), annularity array spot (Fig. 4b). The corresponding phase maps were given as shown in Figs. 3a and 4a. The number of sampling cells of $5 \times 5$ array is $16 \times 16$, and the number of sampling cells of the annularity array is $32 \times 32$. The uniformity of the gratings designed is less than $5 \%$. And the diffractive efficiency is $78 \%$ and $73 \%$, respectively, which are about $10 \%$ higher than the efficiency of Dammann grating.

\subsection{The fabrication of the inseparable binary-phase gratings}

Take the $7 \times 7$ array element for an example to give an illustration of the fabrication of the binary-phase gratings. The diameter of the element fabricated is $50 \mathrm{~mm}$, and the length of a single period is $266 \mu \mathrm{m}$. With the wavelength $\lambda=1.064 \mu \mathrm{m}$, and the focal length of the lens $f=150 \mathrm{~mm}$, the interval of the adjacent spot of the shaped beam is $\Delta=\lambda f / d=0.6 \mathrm{~mm}$. The diameter of the single spot of the shaped focal spot is $d=0.45 \mathrm{~mm}$.

The very large-scale integration (VLSI) technique [15] was adopted in our experiment to fabricate the binary-phase gratings. The first step is to make the mask with electron-beam writing equipment. A thin layer of photo-resist is spun onto a glass substrate. With ordinary microelectronic lithography technology, the mask pattern is transformed into a photo-resist layer upon a glass substrate. The inductive coupled plasma technique was used to transfer the photo-resist pattern onto the substrate. The refractive index of the glass that was used at a wavelength of a

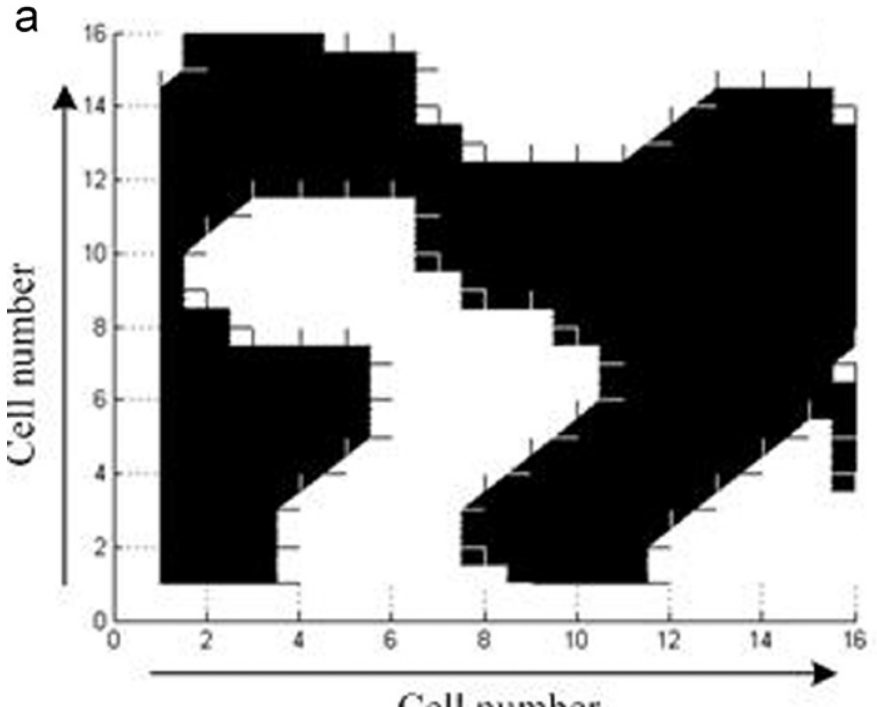

Cell number b

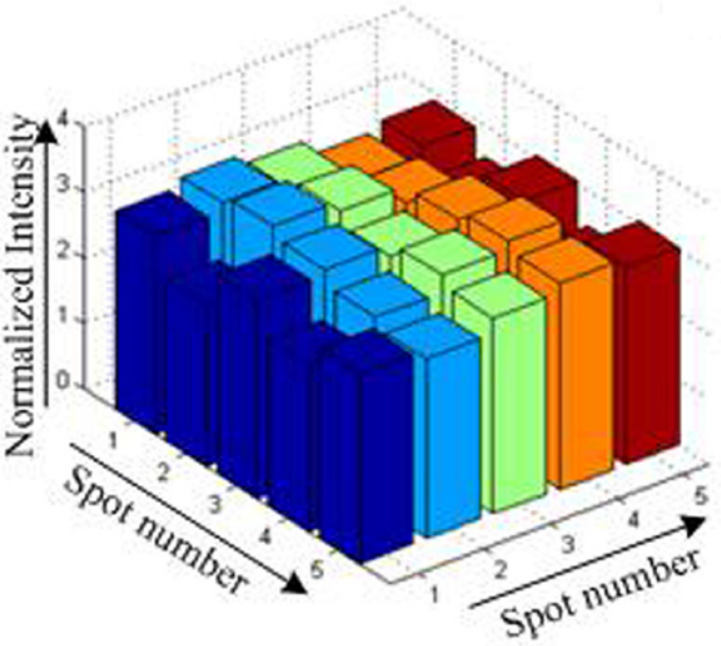

Fig. 3. Designed result of $5 \times 5$ array spot: (a) phase distribution, (b) output intensity profile.
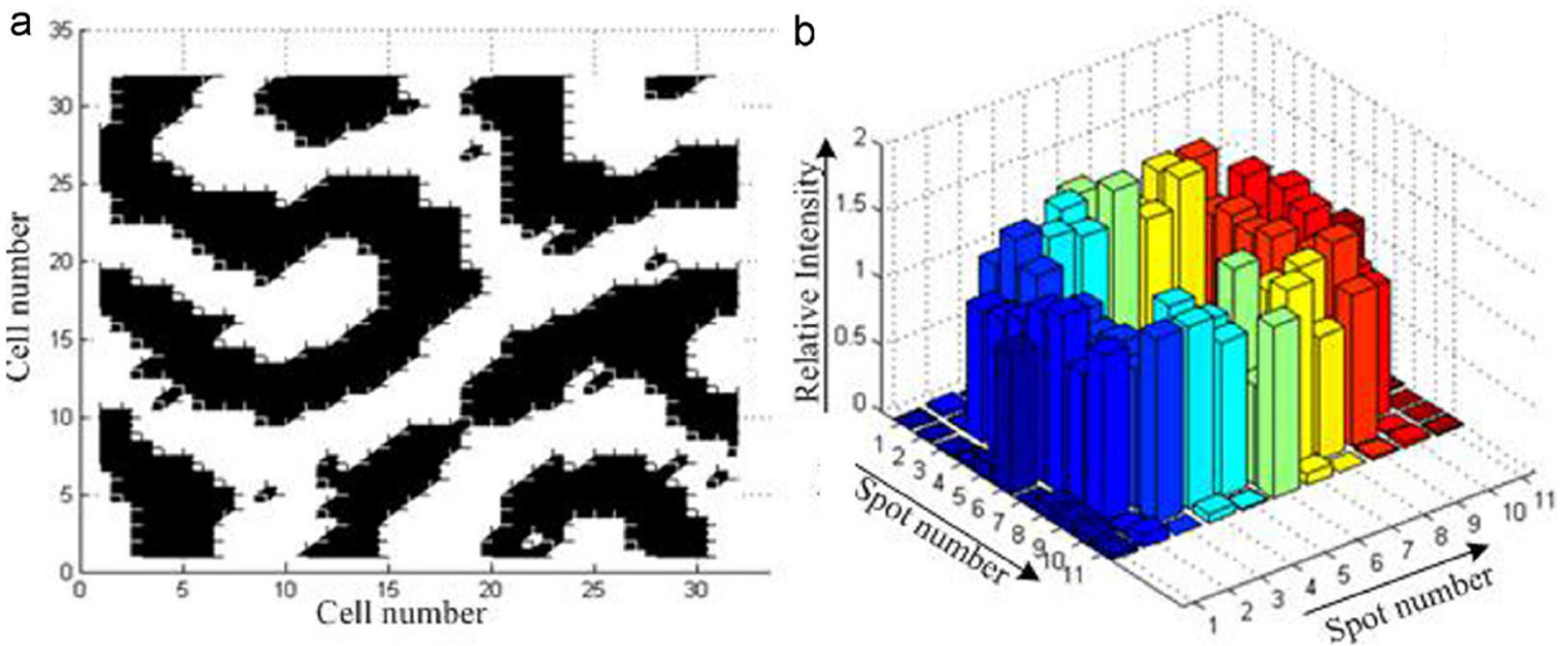

Fig. 4. Designed results of annulus array spot: (a) phase distribution, (b) output intensity profile. 


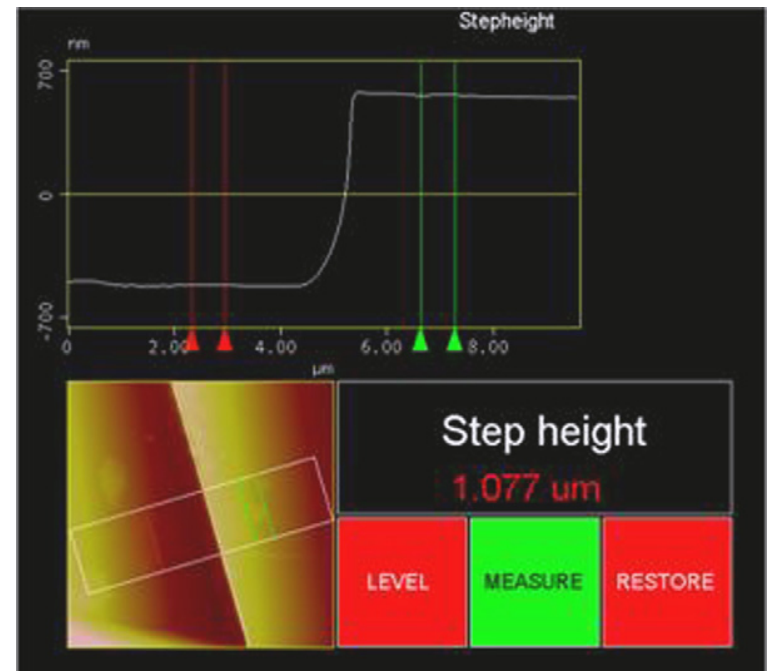

Fig. 5. Surface etching profile of the fabricated $7 \times 7$ array binary-phase grating.

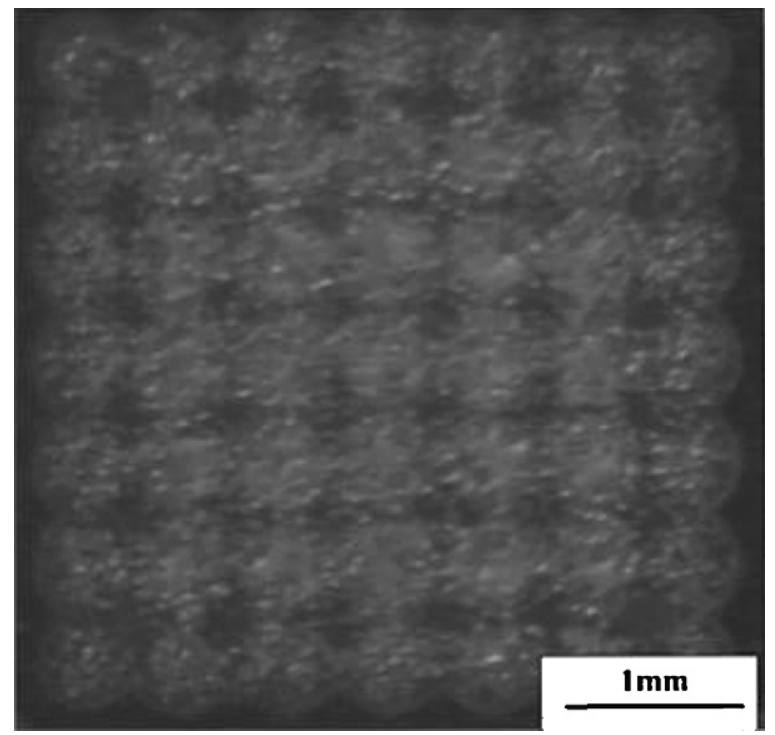

Fig. 6. Surface morphology of ductile iron hardened by $7 \times 7$ array of laser beam.
$1.064 \mu \mathrm{m}$ was $n=1.5067$, and the thickness corresponding to the phase difference $\pi$ is $1046 \mathrm{~nm}$. The surface profile of a fabricated $7 \times 7$ array grating measured by scanning probe microscopy is shown in Fig. 5. It can be seen that the average depth of this surface-relief element is $1077 \mathrm{~nm}$, slightly deviating from the desired value. The fabrication error is less than $5 \%$. The small phase and position errors have almost no effect on efficiency but have a small effect on the uniformity, according to our numerical simulations.

\section{The application of inseparable binary-phase gratings in surface hardening process}

The as-cast pearlitic-ferrite matrix ductile irons, with the nominal chemical composition (at\%) of $3.4 \mathrm{C}, 2.19 \mathrm{Si}, 0.60 \mathrm{Mn}$, $0.018 \mathrm{~S}, 0.040 \mathrm{P}$ and balance $\mathrm{Fe}$, were selected as the tested material. The ductile iron specimens, $12.5 \mathrm{~mm} \times 12.5 \mathrm{~mm} \times 19 \mathrm{~mm}$ in size, were laser hardened by a HLD2006D Nd:YAG laser equipped with the designed binary-phase gratings. The pulsed laser processing parameters were as follows: laser output power $2000 \mathrm{~W}$, pulse frequency $2 \mathrm{~Hz}$, pulse duration $15 \mathrm{~ms}$. Two-dimensional array intensity distribution of the employed pulsed laser beam at the focal plane could be acquired after the transformation. Modified by the $7 \times 7$ array laser beam at the focal plane, the surface morphology of ductile iron (Fig. 6) was obtained.

Fig. 7(a) [16] shows optical micrograph of the cross-section of the laser modification layer, it is clearly seen that the layer consists of laser melted thin surface layer, laser transition zone and laser transformation hardened zone. Also, it is interesting that the microstructure of the surface-section of the layer (Fig. 7(b)) [16] is non-uniform because of the effect of a single-spot laser beam with $7 \times 7$ two-dimensional array distribution, and the microstructure distribution is similar as that along depth direction (Fig. 7(a)). Fig. 8 shows the two-dimensional microhardness map of the cross-section of the half-layer modified by the laser with the original Gaussian distribution. It shows that high hardness zone mostly distribute in the bottom of the modified layer with low hardness in the top. This kind of distribution of hardness is not preferred for improving the wear resistance of the modified layer. Fig. 9 is the two-dimensional microhardness map of the cross-section of the laser surface layer modified by the laser beam shaped to $7 \times 7$ two-dimensional array. It clearly shows periodic gradient hardness distribution, which results from the gradient distribution of microstructure.

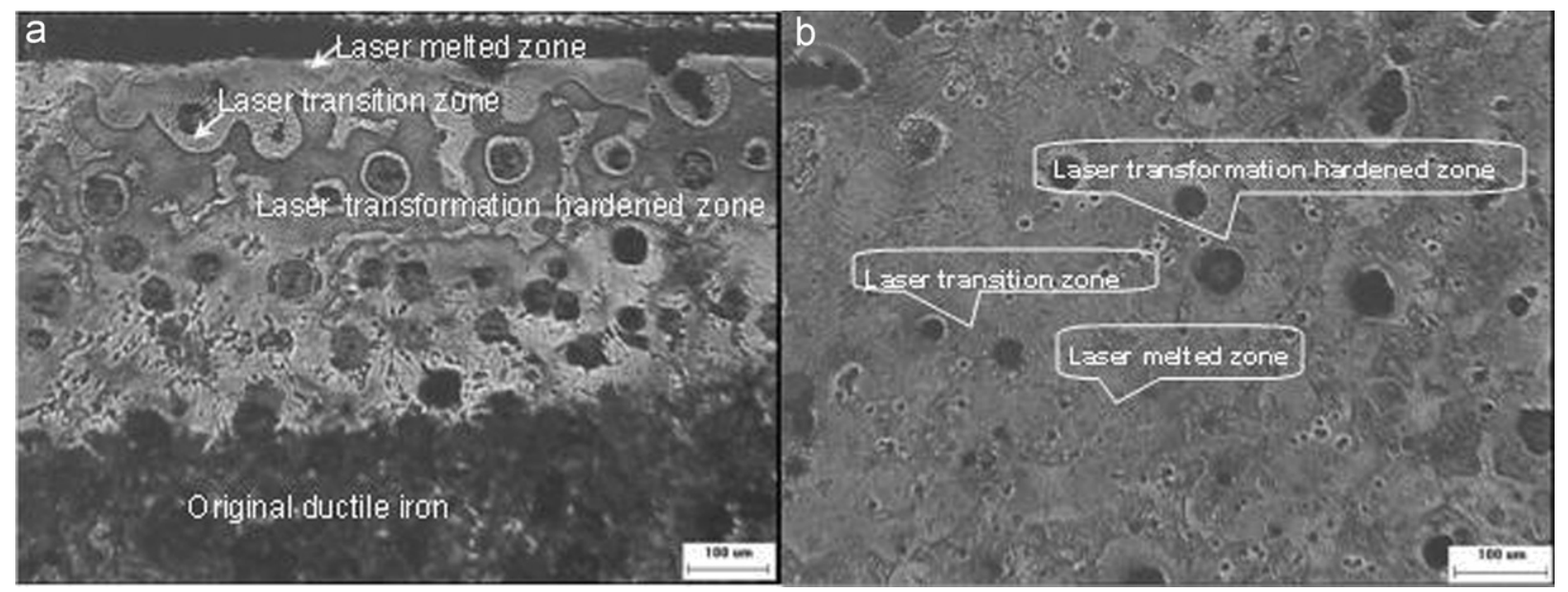

Fig. 7. Optical micrograph showing cross-section microstructure (a) and the surface-section microstructure (b) of laser modified layer. 


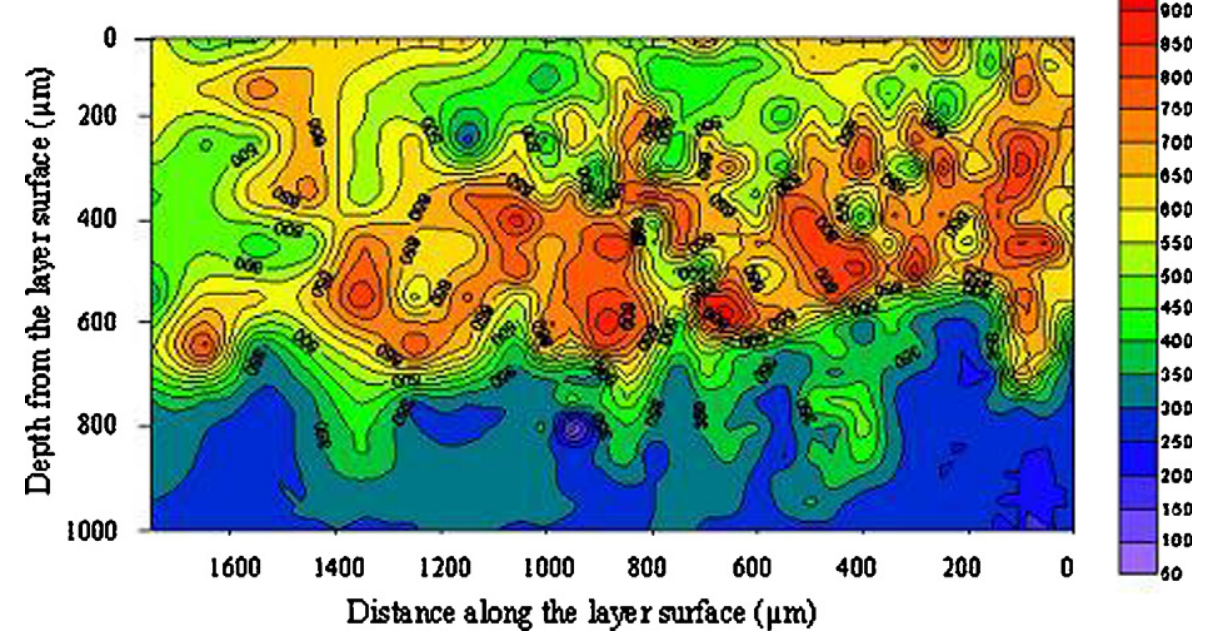

Fig. 8. Two-dimensional microhardness map of pulsed laser surface modified layer. (With the Gaussian laser beam. The parameter of the laser: $2000 \mathrm{~W}, 75 \mathrm{~ms}, 2 \mathrm{~Hz}$ ).

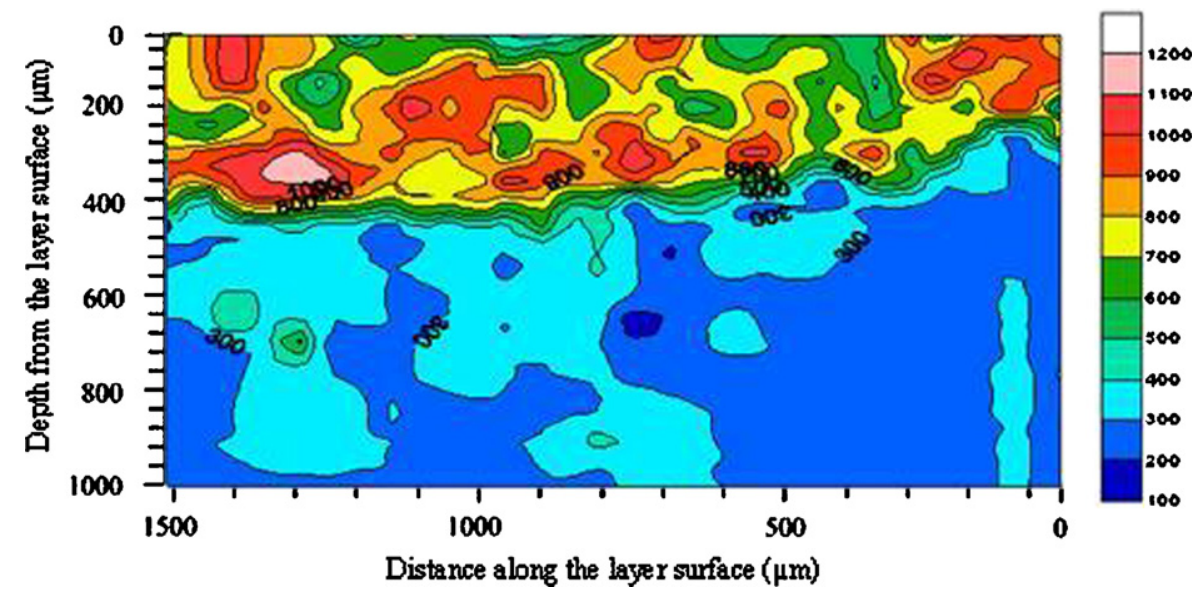

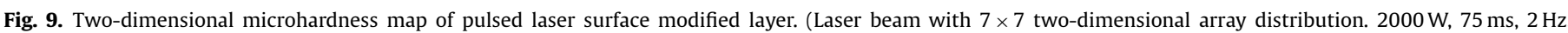
DOE: $7 \times 7$ ).

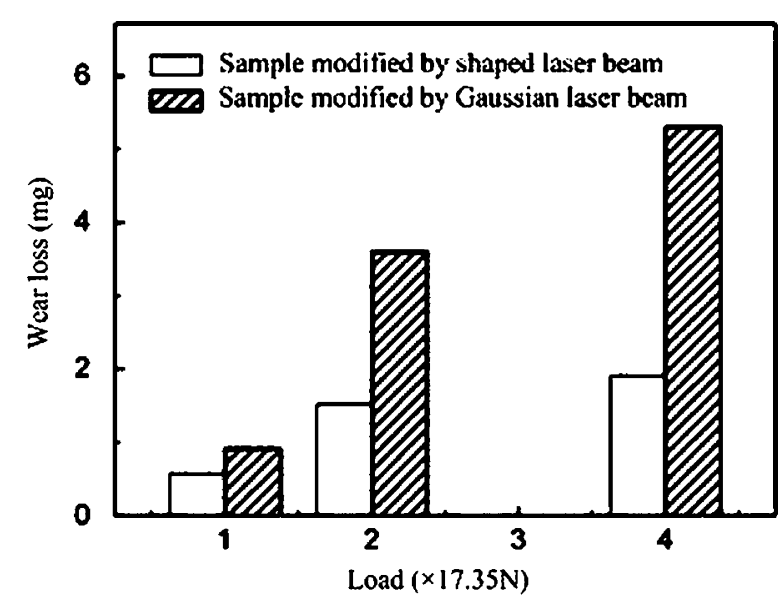

Fig. 10. Mass loss contrast in dry sliding wear testing.

Dry sliding wear testing was taken to contrast the wear resistance property of the surface treated by Gaussian laser and the laser beam with $7 \times 7$ two-dimensional array distribution. The experiments were performed under the ring rotate speed of $40 \mathrm{rpm}$, the time duration of $20 \mathrm{~min}$, and room temperature. The result is shown in Fig. 10. It can be seen that under any load condition, the mass loss of the sample modified by Gaussian laser beam is more than that of the sample modified by the shaped laser beam. With the increase of load, the extent of improvement in wear resistance for the sample modified by the shaped laser beam also increases. When the load is $69.4 \mathrm{~N}$, the wear mass loss of sample modified by laser beam with $7 \times 7$ twodimensional array distribution is 1.5 times less than that of sample modified by Gaussian laser beam. Fig. 11 shows the morphology of worn surface of the two samples. It clearly shows that the worn surface of the sample modified by the shaped laser (Fig. 11b) looks much smoother than that of the sample modified by Gaussian laser beam (Fig. 11a). Compared with the sample treated by conventional Gaussian laser beam, the wear resistance of the ductile iron sample treated by the shaped laser beam improved indeed. Therefore, use of binary-phase gratings is expected to improve the wear resistance of the metal parts under high impact loads bearing conditions and, hence, enhances the service life of the dies.

\section{Conclusions}

The inseparable binary-phase gratings designed and fabricated are applicable for high-power laser beam shaping, and well applied in automobile stamping dies surface modification process. 

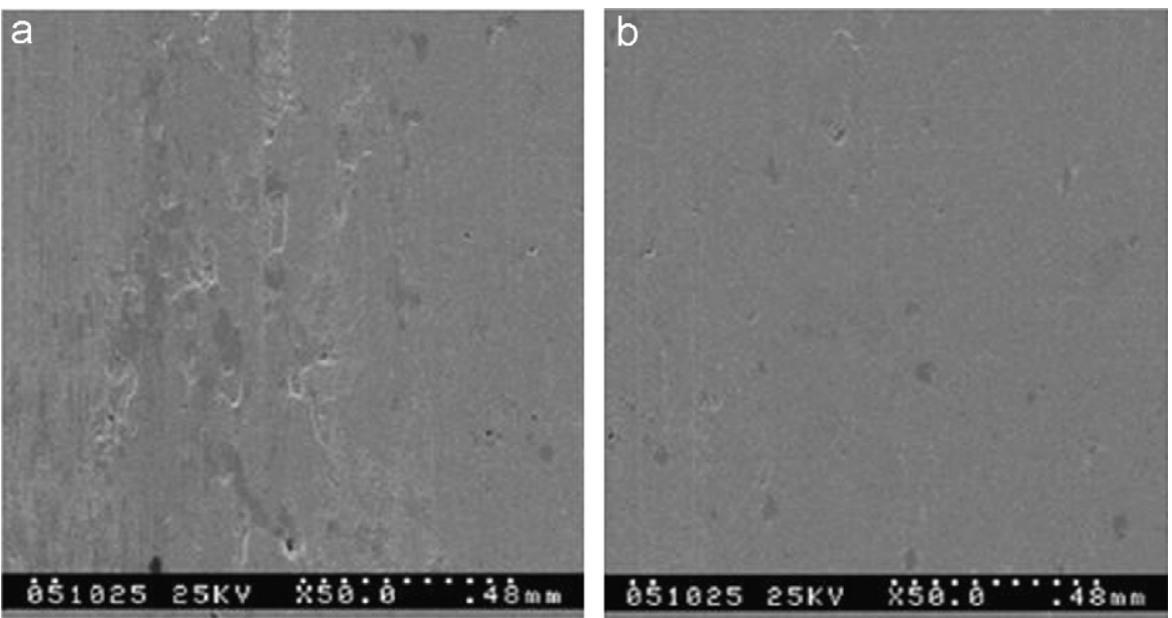

Fig. 11. The morphology of worn surface modified by Gaussian laser beam (a), and by laser beam with $7 \times 7$ two-dimensional array distribution (b).

The adoption of two-dimensional even sampling encode scheme overcomes the limitation of Dammann gratings in two-dimensional design, and the diffractive efficiency is improved. The application of the inseparable binary-phase gratings in surface modification made the ductile iron surface show periodic gradient hardness distribution, which results from the gradient distribution of microstructure. And the wear resistance of ductile iron has improved, which shows prosperous application future.

\section{Acknowledgments}

The authors are grateful for the financial support from the China Postdoctoral Science Foundation (Grant number: 20060400105) and the Foundation of the State Key Laboratory of Nonlinear Mechanics from Institute of Mechanics, Chinese Academy of Science. The invaluable assistance of Dr. Hong-Wei Song, senior engineering Wei-Jian Ning and engineer Li-Xin Wang of the Laboratory for Laser Intelligent Manufacturing, Institute of Mechanics, Chinese Academy of Sciences, during the experiments, are gratefully acknowledged.

\section{References}

[1] Tan QF, Yan YB, Jin GF. Diffractive optical element used to control side-lobe to be extremely low in a large region. Optik 2005;116(10):500-4.

[2] Sanner N, Huot N, Audouard E, Larat C, Huignard JP. Direct ultrafast laser micro-structuring of materials using programmable beam shaping. Opt Lasers Eng 2007;45(6):737-41
[3] Roy A, Manna I. Mathematical modeling of localized melting around graphite nodules during laser surface hardening of austempered ductile iron. Opt Lasers Eng 2000;34:369-83.

[4] Hossack WJ, Darling AM, Dahdouh A. Coordinate transformations with multiple computer-generated optical elements. J Mod Opt 1987;34: 1235-50.

[5] Zhou CH, Jia J, Liu LR. Circular Dammann grating. Opt Lett 2003;28(22): 2174-6.

[6] Zhao S, Chung PS. Design of a circular Dammann grating. Opt Lett 2006;31(16):2387-9.

[7] Taghizadeh MR, Blair P, Balluder K, Waddie AJ, Rudman P, Ross N. Design and fabrication of diffractive elements for laser material processing applications. Opt Lasers Eng 2000;34:289-307.

[8] Tan QF, Yan YB. Composite diffractive optics array element for generating a uniform focal spot. Chin J Lasers 1999;26(9):803-7.

[9] ] Lohmann AW. An array illuminator based on the Talbot-effect. Optik 1988; 79:41-5.

[10] Dammann H, Görter K. High-efficiency in line multiple imaging by means of multiple phase holograms. Opt Commun 1971;3:312-5.

[11] Vasara A, Mohammad RT, Turunen J, Westerholm J, Noponen E, Ichikawa $H$ et al. Binary surface-relief gratings for array illumination in digital optics. Appl Opt 1992;31(17):3320-36.

[12] Yoshikawa N, Itoh M, Yatagai T. Phase optimization of a kinoform by simulated annealing. Appl Opt 1994;33(5):863-8.

[13] Kim MS, Guest CG. Simulated annealing algorithm for binary phase only filters in pattern classification. Appl Opt 1990;29(8):1203-8.

[14] Stephan T, Brigitte S, Thomas B. Efficient design of paraxial diffractive phase elements with descent search methods in Diffractive and Holographic Optics Technology. In: Cindrich I, Lee SH, editors. II SPIE Proceedings, vol. 2404, 1995. p. 40-9.

[15] Margaret BS. Binary optics: A VLSI-based microoptics technology. Microelectron Eng 1996;32:369-88.

[16] Chen Y, Gan CH, Wang LX, Yu G. Laser surface modified ductile iron by pulsed Nd:YAG laser beam with two-dimensional array distribution. Appl Surf Sci 2005;245:316-21. 